\title{
Effects of interpolation methods in spatial normalization of diffusion tensor imaging data on group comparison of fractional anisotropy
}

\author{
Tzu-Cheng Chao ${ }^{\mathrm{a}, \mathrm{b}}$, Ming-Chung $\mathrm{Chou}^{\mathrm{a}, \mathrm{c}}$, Pinchen Yang ${ }^{\mathrm{d}, \mathrm{e}, *}$, \\ Hsiao-Wen Chung ${ }^{\mathrm{a}, \mathrm{c}}$, Ming-Ting $\mathrm{Wu}^{\mathrm{b}, \mathrm{f}}$ \\ ${ }^{a}$ Department of Electrical Engineering, National Taiwan University, Taipei 106, Taiwan \\ ${ }^{\mathrm{b}}$ Department of Radiology, Kaohsiung Veterans General Hospital, Kaohsiung 813, Taiwan \\ ${ }^{\mathrm{c}}$ Department of Radiology, Tri-Service General Hospital, Taipei 100, Taiwan \\ ${ }^{\mathrm{d}}$ Department of Psychiatry, Kaohsiung Medical University, Kaohsiung 807, Taiwan \\ ${ }^{\mathrm{e}}$ Department of Psychiatry, Kaohsiung Medical University Hospital, Kaohsiung 807, Taiwan \\ ${ }^{\mathrm{f}}$ School of Medicine, National Yang-Ming University, Taipei 112, Taiwan \\ Received 7 March 2008; revised 9 September 2008; accepted 25 September 2008
}

\begin{abstract}
This study investigated the effects on the measurement of fractional anisotropy (FA) during interpolation of diffusion tensor images in spatial normalization, which is required for voxel-based statistics. Diffusion tensor imaging data were obtained from nine male patients with attention deficit/hyperactivity disorder and nine age-matched control subjects. Regions of interest were selected from the genu of corpus callosum (GCC) and the right anterior corona radiata (RACR), with FA values measured before and after spatial normalization using two interpolation algorithms: linear and rotationally linear. Computer simulations were performed to verify the experimental findings. Betweengroup difference in FA was observed in the GCC and RACR before spatial normalization $(P<00001)$. Interpolation reduced the measured FA values significantly $(P<00001$ for both algorithms) but did not affect the group difference in the GCC. For the RACR, the between-group difference vanished $(P=.968)$ after linear interpolation but was relatively unaffected by using rotationally linear interpolation $(P=.00001)$. FA histogram analysis and computer simulations confirmed these findings. This work suggests that caution should be exercised in voxel-based group comparisons as spatial normalization may affect the FA value in nonnegligible degrees, particularly in brain areas with predominantly crossing fibers.
\end{abstract}

(C) 2008 Elsevier Inc. All rights reserved.

\section{Introduction}

Diffusion tensor imaging (DTI) using magnetic resonance imaging (MRI) has become a popular noninvasive imaging technique in the examination of microstructures of brain tissues [1-3]. DTI can be used to assess fractional anisotropy (FA) of white matter track in the brain, with lower white matter FA values often indicating alterations in white matter fiber integrity [4]. Studies performing DTI analysis on psychiatric disorders suggested that there may be microstructural changes in the white matter, as reflected by alterations in DTI indices [3]. However, the neurobiological

\footnotetext{
* Corresponding author. Department of Psychiatry, Kaohsiung Medical University Hospital, Kaohsiung 807, Taiwan. Tel.: +886 7 3121101; fax: +88673218309 .

E-mail address: pichya@cc.kmu.edu.tw (P. Yang).
}

changes in the white matter fibers due to psychiatric diseases are usually subtle compared with physiological injuries, causing detection difficulty on single subjects in the presence of finite signal-to-noise ratios $[5,6]$. For psychiatric disorders, therefore, group analysis via intersubject averaging of imaging data now plays an increasingly important role.

Voxel-based statistical (VBS) analysis could be a useful tool in the unveiling of regional structural differences between groups of subjects in an unbiased manner [7]. Before the voxel-based analysis can be performed, the process of spatial normalization consisting of a series of image deformation procedures must be applied to transform the individual brain image onto a standard template. For morphological images, such as T1-weighted images, the normalization algorithm has been well developed, with free shareware programs easily accessible [8-10]. Spatial normalization of DTI, however, is more complicated in 
that the information contained in one single voxel is multidirectional and such parameters as the apparent diffusion coefficient, FA and neuronal fiber orientation have to be preserved. Although there exist abundant reports using voxel-based morphometric analysis of the FA maps [11-13], to the best of our knowledge, no one has shown that spatial normalization on the FA maps could preserve the correct FA values. In addition, a direct application of spatial normalization on the scalar FA maps may not be technically desirable because the essential information for finding anatomical correspondence of the white matter structures, such as tract orientations, would be excluded [6]. Ideally, therefore, spatial normalization of DTI should be performed in a much more sophisticated way than its scalar image counterpart, and, accordingly, direct application of the handy packages to intersubject analysis of DTI data may be prone to errors with misleading interpretations [14-17].

The spatial normalization process for group comparison includes two major steps: image transformation and image interpolation. Image transformation contains four basic transformation processes — scaling, rotating, shearing and translating, with their effects on the diffusion tensor having been reported comprehensively [9,14-17]. It is in general agreement that rotating and shearing cause an alteration of the principal direction of the diffusion tensor, whereas scaling and translating do not affect the shape and orientation of the diffusion tensor $[16,17]$. Hence, only the orientation of a diffusion tensor is changed by the image deformation, leaving all other properties preserved. Image interpolation, on the other hand, is needed when a combination of several diffusion tensors into a single voxel is involved. Tensor interpolation carried out using ordinary linear interpolation has been pointed out to lead to tensor swelling and FA reduction; hence, the interpolation algorithm referred to as affine invariant or rotationally linear interpolation based on eigenvalue computation has been proposed [18,19]. However, even though both the transformation and interpolation procedures used for the diffusion tensor data are much more sophisticated to a great extent than their T1-weighted counterpart, it remains unclear whether the application of these computationally expensive algorithms is absolutely necessary or adequate for psychiatric studies in terms of preservation of DTI indices.

Since the effects of image interpolation on group analysis of DTI data were relatively less documented than DTI transformation, we aimed to investigate the influence of DTI interpolation in this work. We hypothesized that the two interpolation algorithms used in DTI spatial normalization, namely, linear and rotational interpolation, may affect the statistical interpretation of group comparison results in different degrees. For this purpose, we performed a group comparison of the most commonly used DTI index, FA, obtained from patients with attention deficit/hyperactivity disorder (ADHD) and from age-matched healthy control subjects, with intersubject averaging performed via these two interpolation strategies. The influences on FA were examined by VBS and region-of-interest (ROI) analyses. Computer simulations were further performed to provide a theoretical basis to support our hypothesis of possible alterations of the FA values after DTI interpolation.

\section{Materials and methods}

\subsection{Subjects}

The case group was recruited through the outpatient service of the psychiatry department of our hospital. The control group was recruited from the community. Male subjects aged between 12 and 17 years were recruited. Via a semistructured clinical interview, a translated version of the Schedule for Affective Disorders and Schizophrenia for School-Age Children-Epidemiologic Version [20], the psychiatric diagnosis for each subject was ascertained by a child psychiatrist (P.Y.). The case group met Diagnostic and Statistical Manual IV diagnostic criteria for ADHD of the combined subtype, while the control adolescents were determined to be free of any Axis I diagnosis by the same individual who decided on whether potential subjects for the ADHD group met Diagnostic and Statistical Manual IV criteria. The study subjects consisted of 18 adolescents: 9 patients with ADHD (average age of 14 years) and 9 healthy control subjects (average age of 16 years). Possible neurological abnormalities were ruled out by performing three-dimensional gradient-echo T1-weighted imaging and T2-weighted fast spin-echo imaging before the DTI examination, with images interpreted by one experienced radiologist (M.T.W.). The parents of the subjects gave written informed consent after receiving a full explanation of the procedure of the study. This study, including the MRI sequences used, was approved by the institutional review boards of our hospitals.

\subsection{Image acquisition}

The MRI experiments were performed on a 1.5-T system (GE Signa CVi, Milwaukee, WI, USA). For the purpose of morphological evaluation and spatial normalization, threedimensional gradient-echo T1-weighted images (124 slices) were acquired with a voxel size of $0.94 \times 0.94 \times 1.1 \mathrm{~mm}^{3}$, followed by a 15-direction echoplanar imaging (EPI) protocol $(\mathrm{TR} / \mathrm{TE}=10,000 \mathrm{~ms} / 93.4 \mathrm{~ms})$ incorporating twicerefocused spin echo with inherent eddy current compensation [21] for DTI acquisition with a $b$-value of $1000 \mathrm{~s} / \mathrm{mm}^{2}$, as well as one $b=0$ image at two signal averages. Slice thickness was $4.4 \mathrm{~mm}$ with an in-plane resolution of $2.19 \times 2.19 \mathrm{~mm}^{2}$. A total of 33 slices was obtained covering the whole brain.

\subsection{Spatial normalization}

For spatial normalization, DTI transformation was performed, followed by DTI interpolation, as in the case of scalar image normalization. Transformation of DTI was 
achieved according to the procedure described before $[16,17]$ in which the information on alteration of the diffusion tensor was obtained according to the morphological deformation of the original images (Fig. 1). Briefly, the six independent matrix elements of the diffusion tensor were first calculated from the original DTI images that were carefully examined to be free from motion-induced misregistration within each DTI acquisition. The following normalization procedures of the diffusion tensor data were then performed on the six elements, rather than on the original $b=0$ or $b=1000$ images. The $b=0$ EPI images from the DTI image set were co-registered to T1-weighted gradient-echo images to obtain the co-registration matrix, which was subsequently applied to the tensor component coregistration. The original T1-weighted images were then normalized to a $\mathrm{T} 1$ template, from which the normalization parameters were obtained to derive the transformation matrices. White matter segmentation, for later use as a mask, was performed from the normalized T1 images. The deformation parameters in each step were all stored to transform the tensor elements, including alterations of the tensor orientations as well as registration for cerebral morphological consistency. This transformation method has been shown to exhibit no influence on the positive definiteness of the diffusion tensor in that the transformation matrices are orthonormal. Note that although there exist other algorithms for DTI transformation $[6,15]$, the method chosen in our study has the advantages of computational economy and direct compatibility to existing registration software [16,17]. In addition, since the normalization framework for DTI in this study deals with the transformation and interpolation of diffusion tensor components,

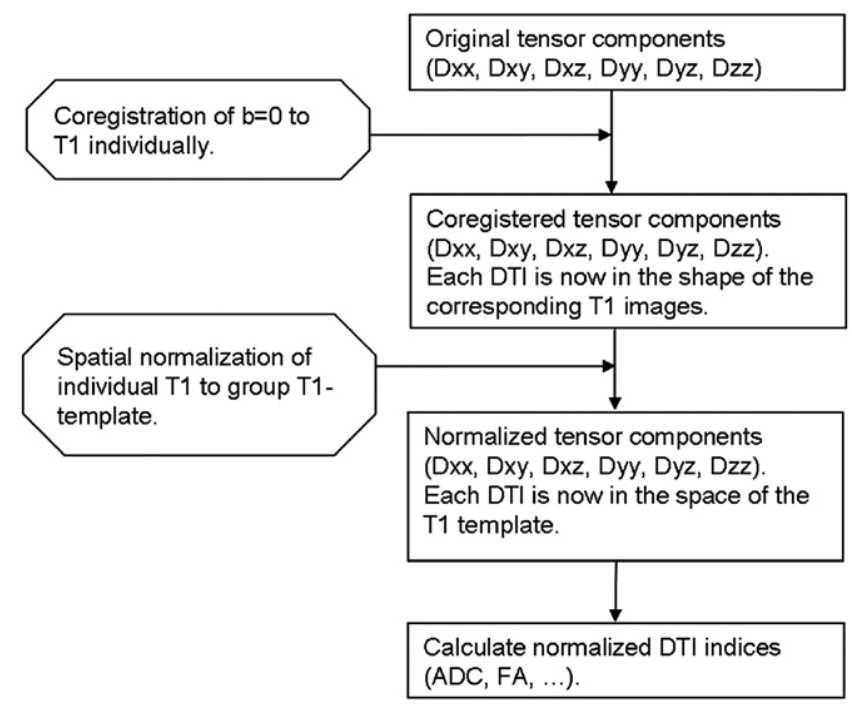

Fig. 1. Flowchart of the procedure for DTI normalization performed based on the scalar image registration algorithm whose information was applied to the additional steps of diffusion tensor transformation and interpolation. The image processing steps are briefly described in the text and detailed in the literature cited. correction for eddy current distortions or motion effects, if necessary, should be performed before calculation of tensor components [22,23].

Following transformation of the diffusion tensor elements to the $\mathrm{T} 1$ template, the pixel from the original image may not be located exactly on the integer pixel grid in the target image space, hence the need for interpolation. For DTI interpolation, two algorithms were separately performed and compared. One was a linear interpolation algorithm that either is available in or can be easily modified from most existing software packages for scalar images [8-10]. The other was a rotationally linear algorithm implemented in-house according to what was described in past reports $[18,19]$. In linear interpolation, weighted averaging of the tensors in the Euclidean space was performed to complete the image normalization, with the weights determined according to the percentage of volume fractions of all the contributing voxels. Linear interpolation is known to preserve the positive definiteness of diffusion tensors, as the method was performed by weighted averaging (where all weights are positive) of positive definite symmetric diffusion tensors. On the other hand, for the rotationally linear interpolation, the procedure involved weighted averaging computations performed on the three eigenvalues of the diffusion tensor. The resultant eigenvalues were incorporated to the three corresponding unit-length eigenvectors whose orientations were modified via linear interpolation. In other words, the interpolations were performed separately for the eigenvalues and for the orientation [18]. Since in this approach the interpolation was performed after solving the eigenvalues, which are all positive, the rotationally linear interpolation also preserves the positive definiteness of the diffusion tensor. In our study, the pixels with negative eigenvalues were ignored, the effect of which on spatial normalization is unknown. Nevertheless, careful retrospective examination showed that these pixels were all located far from the ROIs under the analysis described in subsequent sections and hence did not affect the conclusions drawn from our results.

With the spatial transformation and interpolation procedures stated above, the normalized DTI data were finally rescaled to 3-mm isotropic resolution for subsequent computations of the DTI indices, such as FA. In this manner, spatial normalization of the diffusion tensor data was accomplished. The sets of DTI images from the subjects thus had identical shapes, allowing morphologically independent comparison of all DTI indices on the standard brain template.

\subsection{Data analysis}

There were three types of FA maps generated in this study: two types of group FA maps created by using linear and rotationally linear interpolations (to be used for DTI group analysis) and the 18 sets of individual FA maps generated from the original DTI images for each of the 18 subjects without going through any spatial normalization process. The 18 original individual FA maps were to be used 
for validation, as their FA values were not affected by tensor transformation or interpolation. For the two group maps, FA values were compared using VBS analysis on SPM5. Prior to the analysis, a Gaussian kernel with 6-mm full width at half maximum was applied on the maps after spatial normalization. The use of different filter sizes did not yield significant cluster changes from, for example, the use of 8mm full width at half maximum in our data sets [24,25]. Voxels belonging to the gray matter were excluded by applying the white matter-segmented images on the normalized FA maps as a mask. Regions exhibiting statistically significant group differences between ADHD patients and control subjects were identified as those with the threshold of $P<.01$ and cluster sizes of more than 10 contiguous voxels. After this VBS analysis step, white matter regions showing group differences in FA could be visualized from both the linearly interpolated maps and the rotationally linearly interpolated maps.

Following VBS identification of regions showing FA differences between ADHD patients and healthy control subjects, ROI analysis was also performed using the 18 sets of original FA maps to see whether the group DTI analyses after spatial normalization gave the same results as would be found from the original data without using spatial normalization. For this purpose, two ROIs were chosen from the group FA maps that showed VBS differences between ADHD patients and healthy control subjects. The rationale for choosing these two ROIs is stated in a subsequent section. The selection of these ROIs on the original FA maps was accomplished by one operator (T.C.C.) and confirmed by an experienced radiologist (M.T.W.). Following ROI selection, the FA values were recorded on a pixel-by-pixel basis. As a result, the group comparison for FA between
ADHD patients and control subjects was achieved via three methods: one method with tedious recording of FA values pixel by pixel from every individual on preidentified ROIs and two methods with intersubject spatial normalization using linear and rotationally linear interpolations. Differences in FA values between the ADHD and control groups were compared and reported for the three methods. FA distributions in the form of histograms [26,27] were also generated. Note that since the subjects had different brain volumes, the following steps were also executed to rule out possible volume effects on ROI selection: For all the ROIs selected from the original FA maps, the same spatial normalization procedure was performed on the ROIs, after which the deformed ROIs shown on the normalized FA maps were examined for correctness by the experienced radiologist (M.T.W.) and with the ROI volumes recorded. Statistics were computed using Student's $t$ test (SPSS, Chicago, IL, USA). Results with $P<.05$ (one tailed) were considered statistically significant.

\subsection{Computer simulation}

Simulations were performed to provide theoretical support for our hypothesis of FA alterations after DTI interpolation. Since interpolation of diffusion tensors with different orientations or shapes may have different impacts, their effects were investigated separately. The influence of tensor orientation was examined by mathematically generating two diffusion tensors having identical eigenvalues with a ratio of $5: 1: 1(\mathrm{FA}=0.770)$, at an angle varying from $0^{\circ}$ to $60^{\circ}$ from each other. Interpolation was then performed assuming partial volume ratios varying from 1.0:0.0 to 0.0:1.0 at 0.1 stepping (e.g., 0.9:0.1, 0.8:0.2, 0.7:0.3, ..., etc.). The
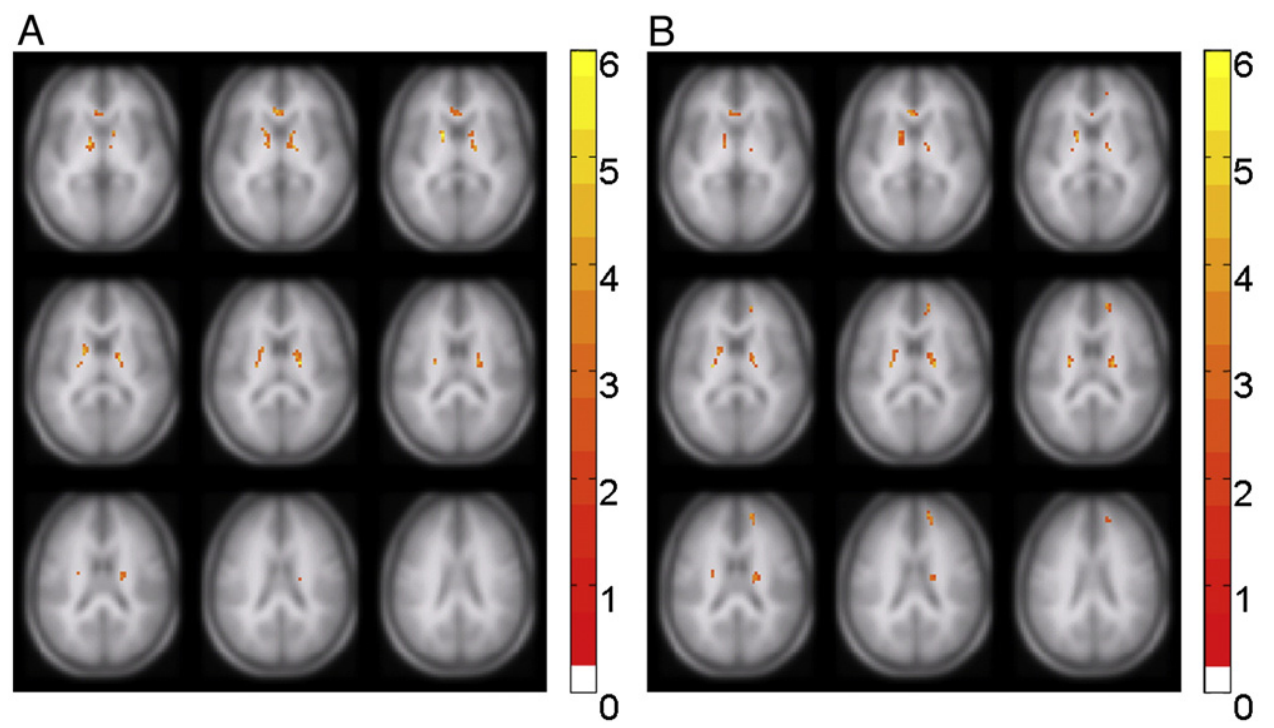

B

Fig. 2. Regions showing statistically significant differences between the ADHD patients and the age-matched healthy control subjects, as obtained via VBS analysis using (A) linear interpolation and (B) rotationally linear interpolation (neurological coordinate). Note that more regions were identified in (B) than in (A). The color bars stand for the $T$ score from two-sample Student's $t$ test. 
resulting changes in FA values after interpolation were then plotted for both the ordinary linear and rotationally linear interpolation algorithms.

The effects of shape differences of the diffusion tensors were simulated with interpolation performed on two tensors mathematically created with different geometries, while their principal eigenvectors were aligned in parallel. Specifically, one tensor was given a fixed geometry and then interpolated with another diffusion tensor with varying eigenvalue ratios. Partial volume ratios were again varied as mentioned previously, with resulting FA values plotted. In contrast to the previous simulation that investigated the effects of orientation difference occurring in the presence of fiber crossing, this part of simulation on shape difference with identical principal eigenvectors mainly examined the situation in parallel fiber bundles.

Possible influences on the statistical inference of interpolation-related changes in the shape of diffusion tensors were further demonstrated in the following manner simulating the group comparison process. First of all, diffusion tensors were mathematically generated, with eigenvalue ratios chosen as the typical values found in specific ROIs from the ADHD patients and control subjects. Then, two diffusion tensors with identical shapes but different orientations were interpolated. The change of orientation was carried out by rotating one of the originally aligned tensors along the shortest eigenvector at a designated angle. The abovementioned process was repeated for many combinations of orientations spanning a prespecified angular dispersion, where the angular dispersion was determined according to the presence of fiber crossings: For nearly parallel fiber bundles, a small angular dispersion was assumed for the interpolation, whereas for regions with crossing fibers, a relatively larger angular dispersion was used in the simulation. The resulting effects on group comparison of FA values between simulated ADHD patients and control subjects were then examined. Only linear interpolation was taken into account in this part of the simulation as no change in FA would be found if using rotationally linear interpolation.

\section{Results}

\subsection{VBS analysis}

Panels (A) and (B) of Fig. 2 show the between-group FA differences obtained from the VBS analysis results using linear interpolation and rotationally linear interpolation, respectively. The genu of corpus callosum (GCC) and the internal capsule were found in Fig. 2A and B to exhibit statistically significant reduced FA in ADHD patients compared with healthy control subjects. The right anterior corona radiata (RACR), however, showed FA difference when analyzed with rotationally linear interpolation but not with ordinary linear interpolation under the same statistical threshold.
Since the VBS analysis results using SPM5 suggested FA reductions in ADHD in inconsistent regions for the two interpolation algorithms, we performed further examinations on the FA value distributions using ROI analysis. For this purpose, two ROIs around the GCC and RACR were selected according to Fig. 2 to represent consistent and inconsistent findings on FA difference, respectively. The selection of ROIs is shown in Fig. 3. The numbers of voxels included in the GCC as shown on the normalized images were $38.6 \pm 11.4$ (mean \pm S.D.) for healthy control subjects and $38.4 \pm 10.9$ for ADHD patients. Likewise, for the RACR, the numbers of voxels included were $77.6 \pm 11.4$ for healthy control subjects and 78.6 \pm 10.9 for ADHD patients on the normalized images. The ROI volumes for the GCC and RACR showed no statistical difference between the two groups. Difference in FA, reported below, is therefore unlikely to arise from volume effects during ROI selection. The comparison of interpolation effects on FA against ROI analysis results treated as true values was thus performed on the two ROIs chosen above.

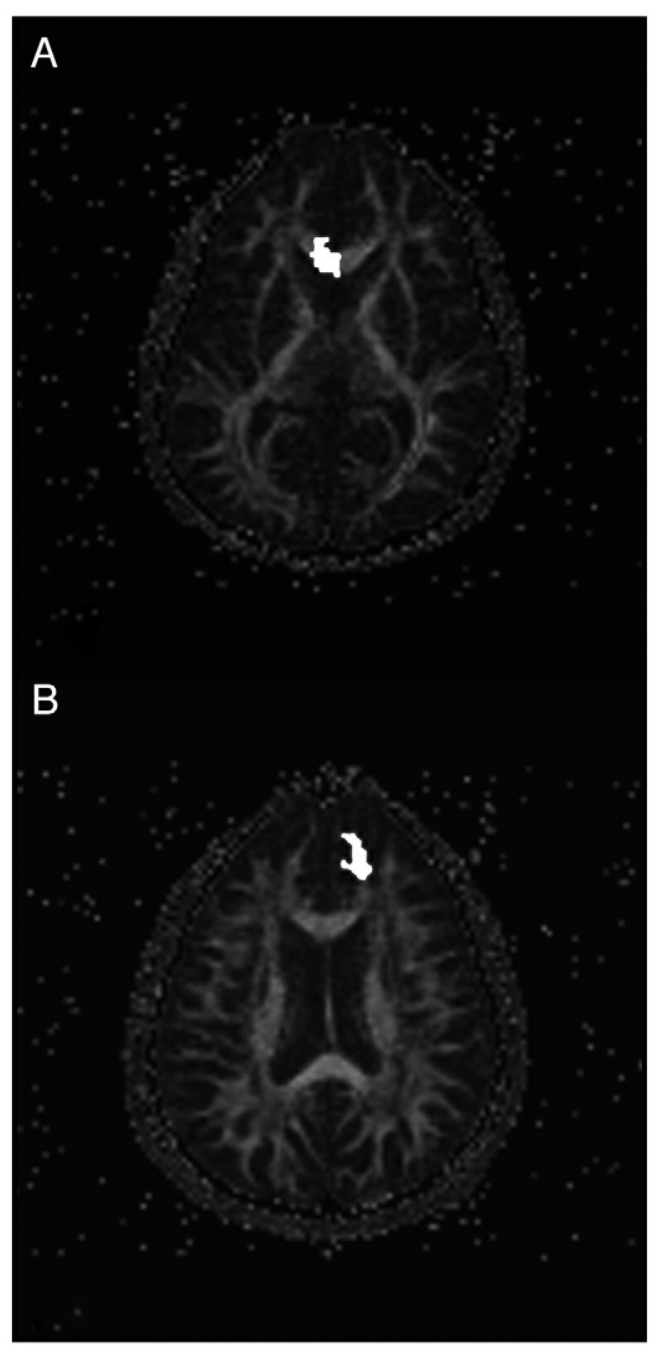

Fig. 3. FA maps showing the selection of ROIs from the (A) GCC and (B) RACR (neurological coordinate) 


\subsection{Comparison against ROI analysis}

Fig. 4 shows the effects of different interpolation algorithms on the group mean values of FA for the GCC (Fig. 4A) and RACR (Fig. 4B). The FA values measured from the original data without spatial normalization were smaller in ADHD patients than in healthy control subjects for both the GCC and RACR ( $P<00001$ for both). After spatial normalization, the FA values for ADHD patients were still found to be significantly smaller than those for healthy control subjects in the GCC regardless of the interpolation method used (Student's $t$ test, $P<00001$ ). In the RACR, however, significant between-group difference in FA in the original images $(0.387 \pm 0.005$ for healthy control subjects and $0.368 \pm 0.002$ for ADHD patients; $P<00001)$ was lost after spatial normalization with linear interpolation $(0.314$ \pm 0.008 for healthy control subjects and $0.314 \pm 0.014$ for ADHD patients; $P=.968$ ). Rotationally linear interpolation, on the other hand, did not change the significant difference
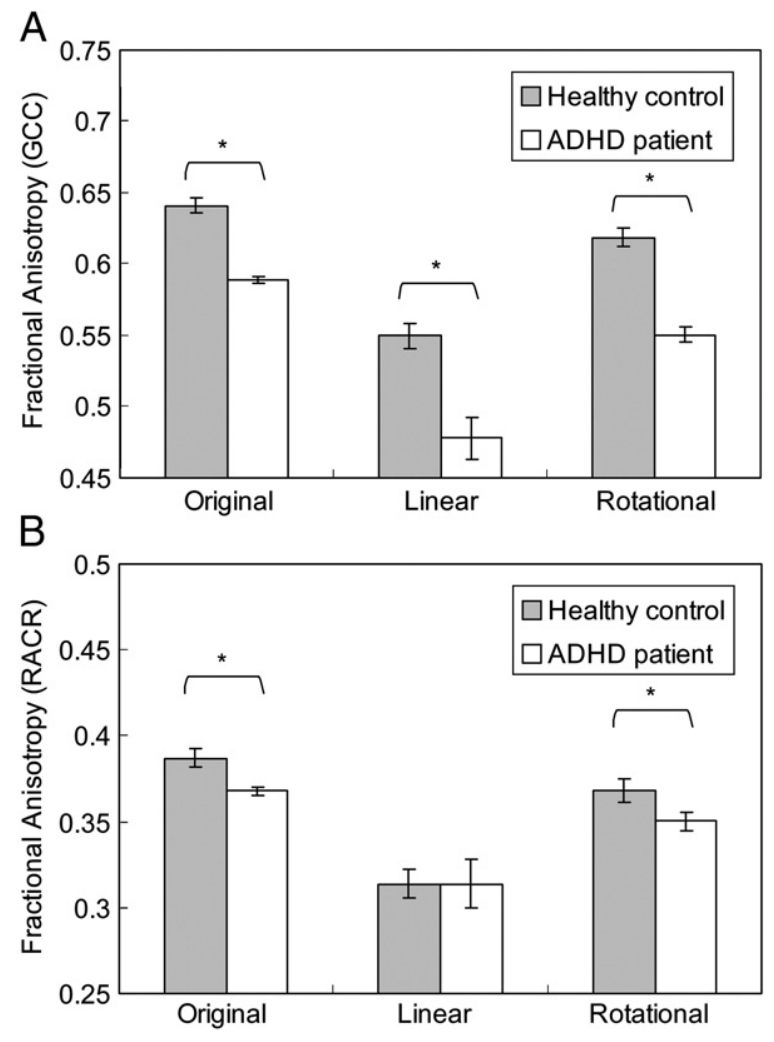

Fig. 4. (A) Means and standard deviations of FA values within the GCC before spatial normalization (left), after normalization with linear interpolation (middle) and after normalization with rotationally linear interpolation (right). Asterisks indicate statistically significant difference between patients with ADHD (white) and healthy control subjects (gray). The group difference in FA was found with $P<00001$ regardless of the interpolation procedure, even with the presence of FA reduction after spatial normalization. (B) Plot for the RACR arranged in a similar manner. Statistically significant difference between ADHD patients and healthy control subjects was found from the original data and those with rotationally linear interpolation $(P<00001)$, but not from those with linear interpolation $(P=.968)$.
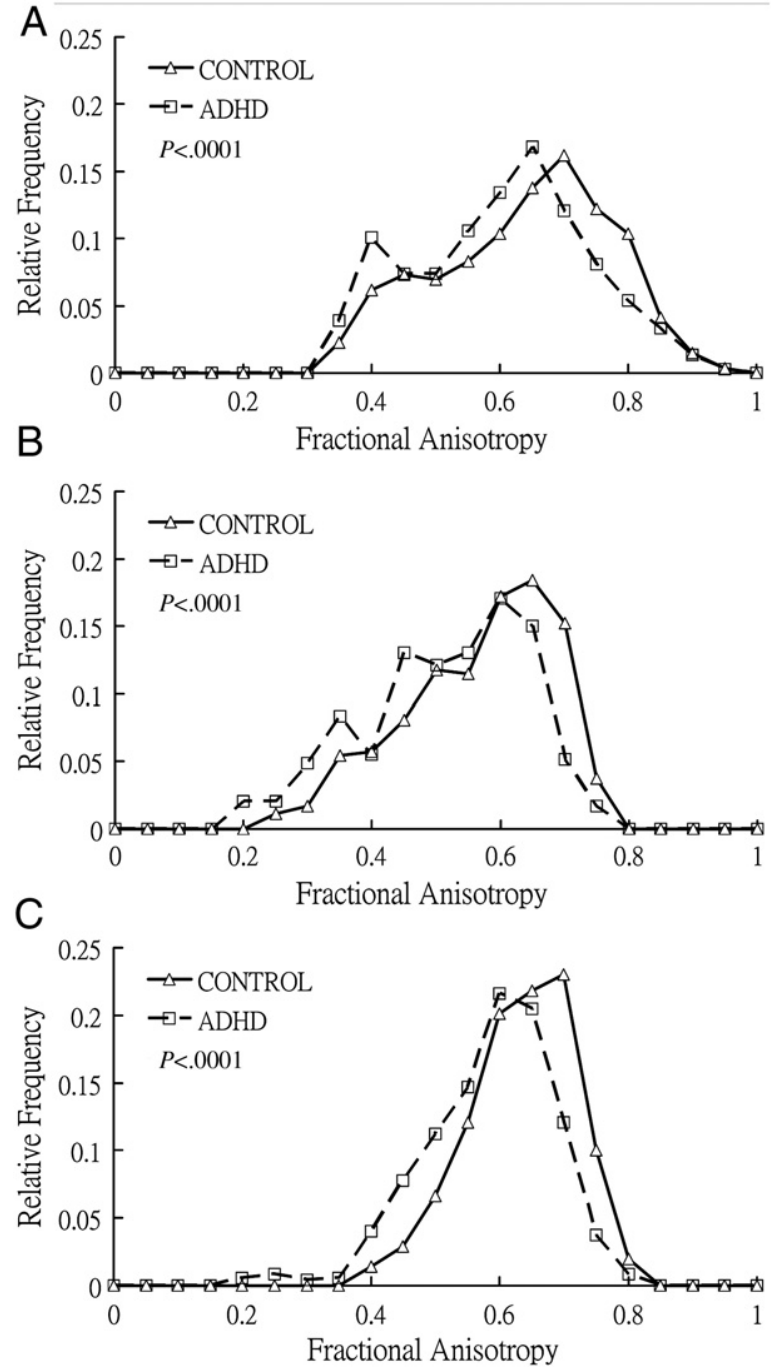

Fig. 5. Histograms of FA distribution within the GCC derived from the (A) original imaging data, (B) FA maps after normalization with linear interpolation and (C) FA maps after normalization with rotational linear interpolation. Linear interpolation is seen to result in a leftward shift of the FA histogram to a greater extent than in rotationally linear interpolation. In addition, a narrowing of the FA distribution is also visually perceivable. Statistical significance for group difference exists in all three situations at a $P<0001$ level.

between groups $(0.368 \pm 0.007$ for healthy control subjects and $0.350 \pm 0.005$ for ADHD patients; $P<00001$ ).

Similar findings were noted for the FA distribution histograms. Fig. 5 shows the FA histograms within the GCC of the ADHD and control groups as measured from the original images (Fig. 5A), the spatially normalized maps using linear interpolation (Fig. 5B) and the spatially normalized maps using rotationally linear interpolation (Fig. 5C). As the figure demonstrates, spatial normalization resulted in a "leftward-shift" and "narrowing" (i.e., concentrated in the middle) pattern of the FA distribution histogram with overall decrease in FA values, but the interpretation of the between-group difference (i.e., significantly smaller FA in ADHD patients; $P<0001)$ remained unchanged for the 


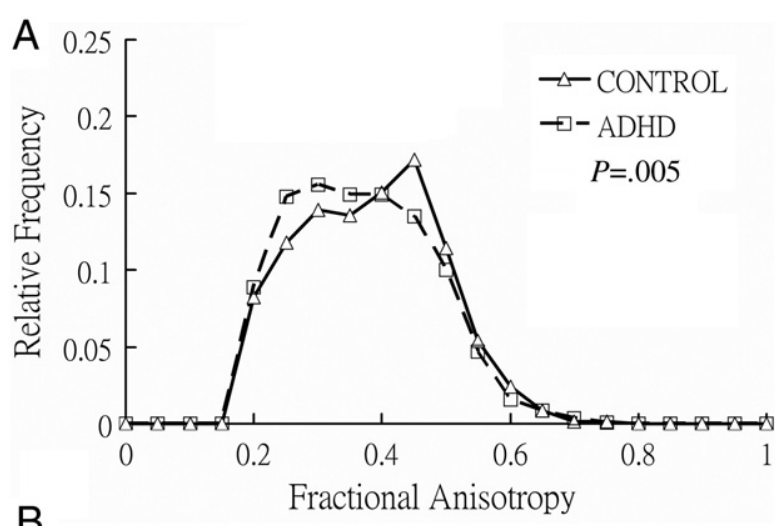

B
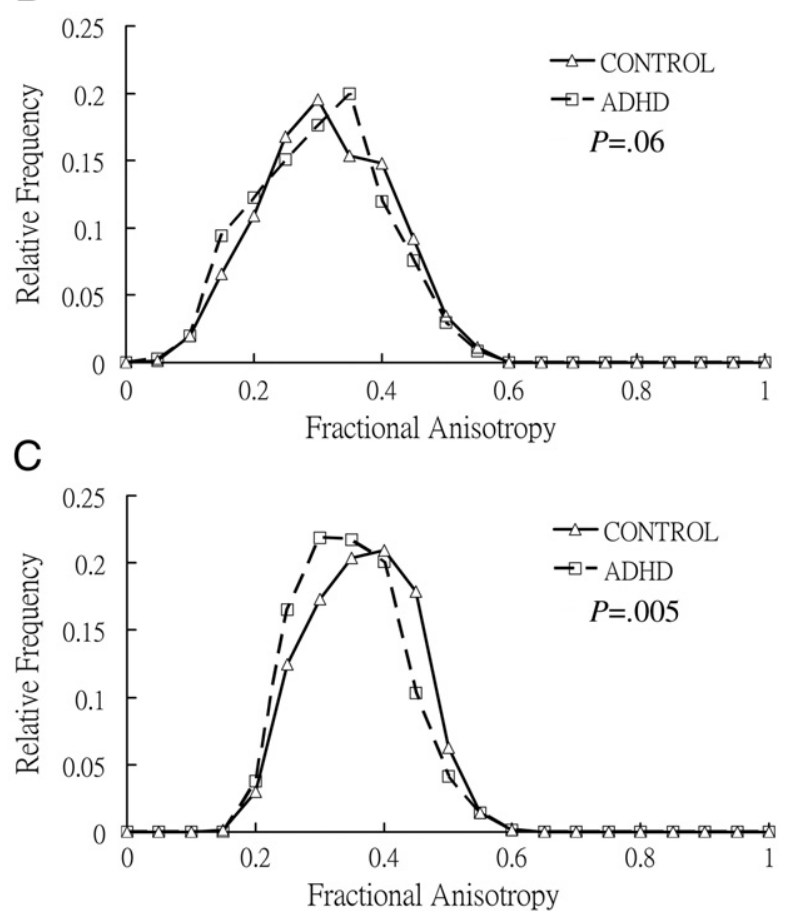

Fig. 6. FA histograms within the RACR arranged in the same manner as in Fig. 5. The FA distributions were significantly different at a $P<.005$ level between ADHD patients and healthy control subjects in both the original data and the spatially normalized data using rotational interpolation. With linear interpolation, however, ADHD patients and healthy control subjects failed to demonstrate any statistically significant difference $(P=.06)$.

GCC. Fig. 6 shows the FA histograms within the RACR in the three aforementioned conditions. There were betweengroup differences noted in the original images $(P<005)$ and in the images after application of the rotationally linear interpolation $(P<005)$. However, these between-group differences disappeared after the application of linear interpolation $(P=.06)$.

\subsection{Simulation results}

The FA reduction effects following image interpolation are further illustrated in the computer simulations. Fig. 7 shows the shapes and orientations of the diffusion tensors obtained from interpolation of two identically shaped diffusion tensors $(\mathrm{FA}=0.770)$ differing in the orientation by an angle of $60^{\circ}$. The partial volume fractions were changed to see the resulting effects. Linear interpolation was found to cause a swelling of the interpolated diffusion tensor and a consequent decrease in FA with prominent partial volume effects, suggesting distortion of FA information, particularly in the presence of fiber crossing. With rotationally linear interpolation, the shape of the diffusion tensor was retained regardless of the partial volume fraction. Quantitative preservation of the FA values with rotationally linear interpolation compared with ordinary linear interpolation is shown graphically in Fig. 7B.

Interpolation of two parallel tensors with different geometries showed varying degrees of FA reduction. Fig. 8 shows the results when one tensor was fixed at an eigenvalue ratio of 4:2:1 $(\mathrm{FA}=0.577)$ and interpolated with three other diffusion tensors having eigenvalue ratios of 4.0:1.5:1.5 (FA=0.552; Fig. 8A), 3:3:1 (FA=0.458; Fig. 8B) and 3:2:2 $(\mathrm{FA}=0.248$; Fig. $8 \mathrm{C}$ ). Both ordinary linear and rotationally linear interpolations could lead to FA decrease, albeit to exactly identical degrees [18]. For most cases such as those shown in Fig. 8A and B, interpolation led to concaved curves, meaning that the resulting FA values were smaller than what would be expected in an ideal situation. The slightly convex curve in Fig. 8C suggested that, in some cases, interpolation did not necessarily cause overall reduction in FA distribution.

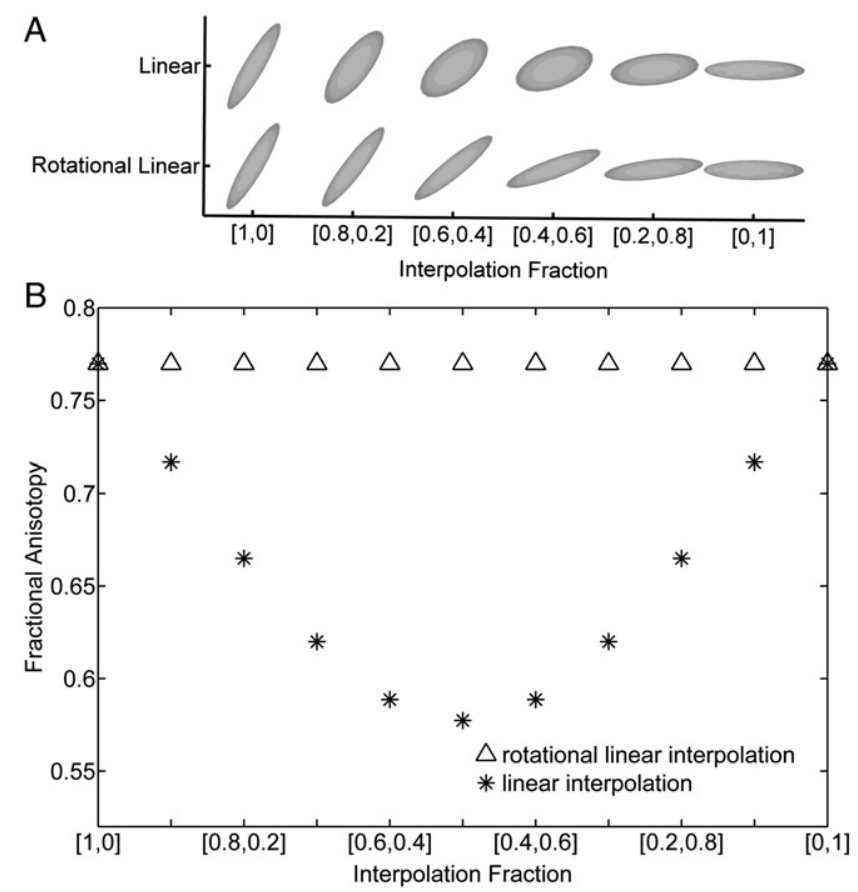

Fig. 7. (A) Graph showing the effects after interpolating two identical diffusion tensors with an angular difference of $60^{\circ}$ (top row, linear interpolation; bottom row, rotationally linear interpolation). The interpolated tensor is seen to swell in the presence of partial volume averaging using linear interpolation, whereas the rotational linear interpolation retains the original shape of the diffusion tensor. (B) FA values of the interpolated tensors plotted at different partial volume fractions demonstrating the same phenomena quantitatively for the two interpolation methods. 
The effects of linear interpolation on the statistical inferences, simulating the situations of the GCC and RACR for ADHD patients and control subjects, are shown
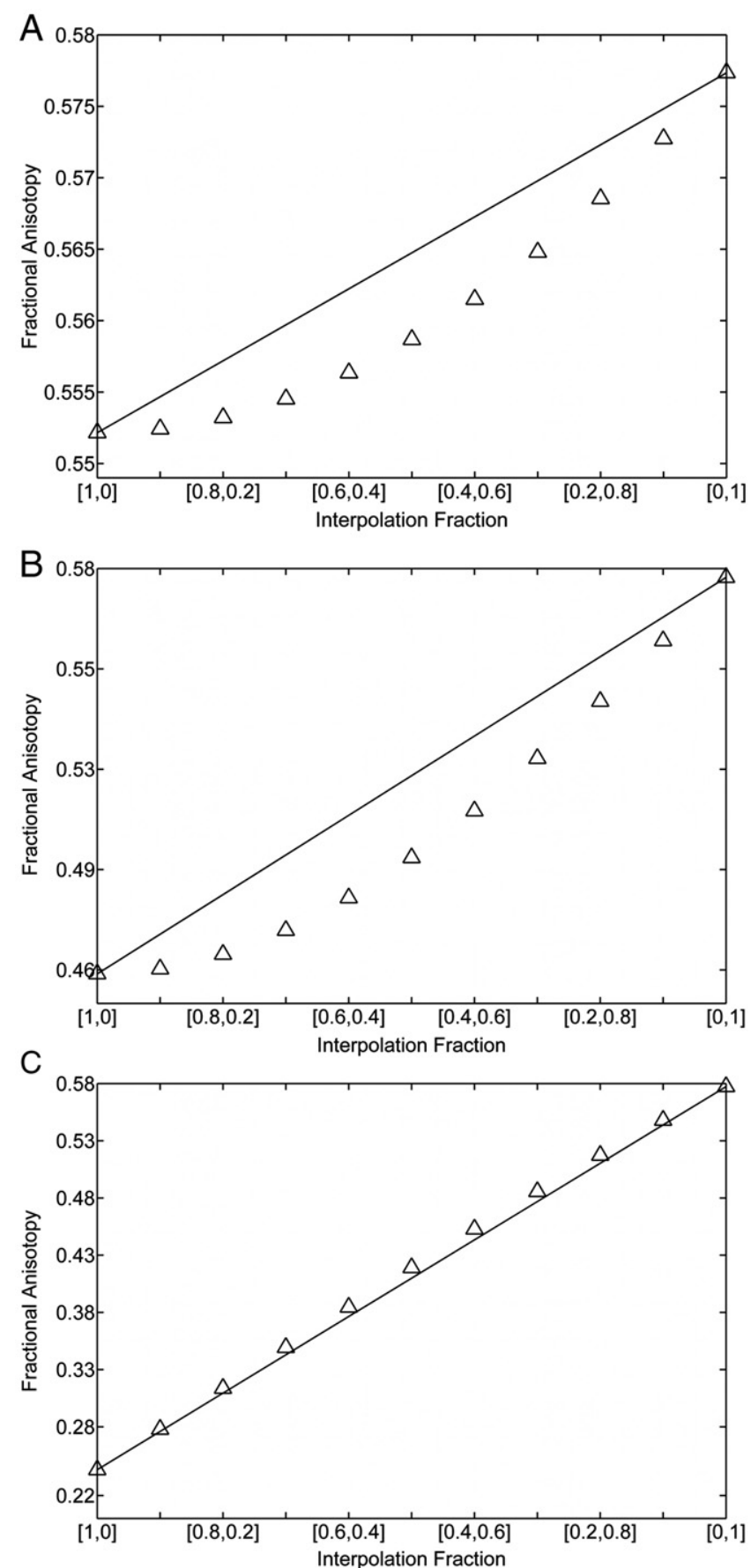

Fig. 8. Examples demonstrating FA reductions through interpolation of two parallel diffusion tensors with different shapes. Simulations were performed by fixing one tensor at $\mathrm{FA}=0.577$ and interpolated with the other tensor at (A) $\mathrm{FA}=0.552$, (B) $\mathrm{FA}=0.458$ and (C) $\mathrm{FA}=0.248$. Ideally, preservation of mean FA in a large ROI is achieved if the interpolated diffusion tensor assumes the FA value as the average weighted by corresponding partial volume fractions (i.e., solid line). However, a concaved behavior was found in most cases, as shown in (A) and (B). A slightly convex curve as in (C) was also observed occasionally. Data from ordinary linear interpolation and rotationally linear interpolation overlapped exactly without difference.
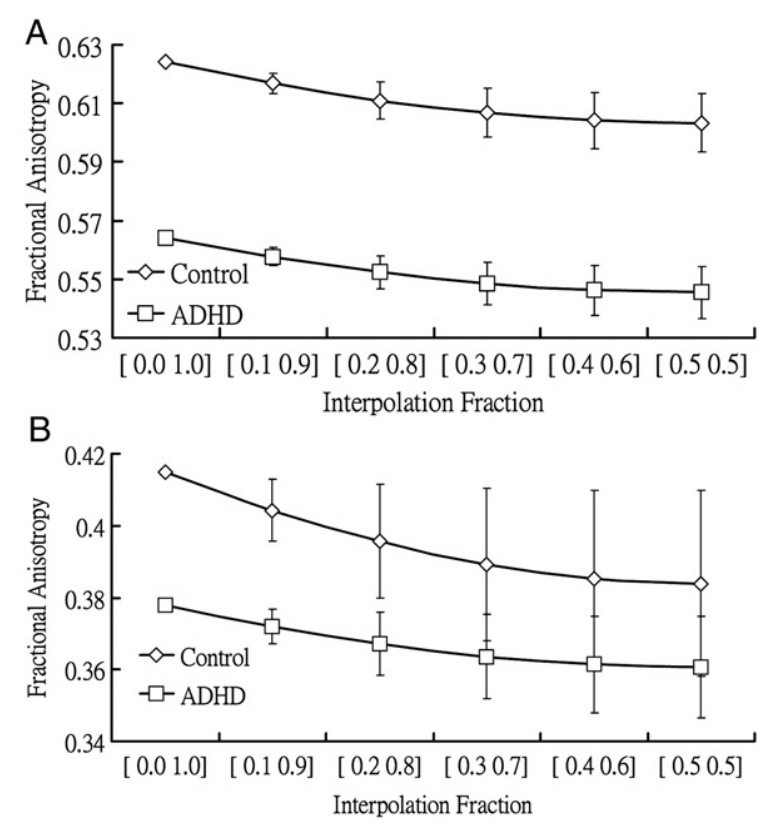

Fig. 9. Potential effects of linear interpolation on the statistical inferences in the presence of nearly parallel fibers and crossing fibers. Group differences in FA are shown when interpolation was performed with (A) angular dispersion up to $25^{\circ}$, simulating the situation of the GCC, and (B) angular dispersion up to $45^{\circ}$, simulating the situation of the anterior corona radiata. Note the reduction in FA difference and the simultaneous increase in the standard deviations as the interpolation fraction increases, which is particularly prominent for the crossing fibers in (B).

in Fig. 9. According to what were found from the subjects included in this study, the FA values for the GCC were set to be 0.624 (eigenvalue ratio=15:5:4.5) and 0.564 (eigenvalue ratio=15:6:5) for control subjects and ADHD patients, respectively, with angular dispersion ranging randomly from $15^{\circ}$ to $25^{\circ}$. As seen from Fig. 9A, the group differences in FA are still preserved between ADHD patients and control subjects for the GCC where the fiber bundles are nearly parallel. On the other hand, for the RACR, the FA values were set to be 0.415 (eigenvalue ratio=9:5:4) and 0.378 (eigenvalue ratio=9:6:4) for control subjects and ADHD patients, respectively, with angular dispersion ranging from $15^{\circ}$ to $45^{\circ}$ due to the presence of fiber crossing in the RACR. The significance in the difference of FA between ADHD patients and control subjects is seen to decrease with linear interpolation (Fig. 9B), due to reduced difference in mean FA as well as increased standard deviation in the presence of large angular dispersion from fiber crossing. These findings confirm that linear interpolation may lead to potential disappearance of group difference in FA, particularly for regions with crossing fibers.

\section{Discussion and conclusions}

Voxel-based intersubject averaging for DTI data is gaining increasing attention from investigators involved in psychiatric studies. Previous reports have documented 
technical influences, such as different filter sizes of the smoothing kernel, on the VBS analysis results of DTI data $[24,25]$. In this work, we further showed that use of different interpolation algorithms could result in distinct group interpretations even with the same smoothing kernel. Our Fig. 2A suggests that, when using linear interpolation, the RACR shows no statistically significant group difference in FA between ADHD patients and age-matched control subjects. Fig. 2B analyzed using rotationally linear interpolation, however, suggests significantly reduced FA in the RACR for ADHD patients. The ROI and FA histogram analyses (Figs. 4 and 6) performed on the original undistorted images confirm the findings of reduced FA as obtained with the rotationally linear interpolation, hence indicating possible information loss during the ordinary linear interpolation procedure.

It is also worth mentioning that the discrepancy between the group comparison results from the two interpolation algorithms as shown for the RACR was not found for the GCC (Fig. 5). Such behavior is believed to be due to differences in their internal neural fiber architecture. The GCC largely contains parallel fibers; hence, the principal eigenvectors associated with diffusion tensor matrix are roughly oriented in the same direction. Interpolation of two tensors in parallel has little influence on the FA value, as demonstrated in the computer simulation results on fiber orientation (Fig. 7). As a consequence, use of ordinary linear interpolation or rotationally linear interpolation does not exhibit different results for the GCC. The internal capsule that exhibits largely parallel fiber bundles shows behavior similar to the GCC (VBS analysis results in Fig. 2; ROI data not shown), consistent with our inference. On the other hand, the RACR substantially consists of crossing fibers, due to which the interpolation is performed on tensors oriented in different directions. Since linear interpolation leads to decreased FA in the presence of fiber orientation difference as shown in the simulation results (Fig. 7), the consequent failure in preserving the FA value could have obscured the between-group difference after spatial normalization, as further demonstrated in computer simulations (Fig. 9). Note that Figs. 7 and 9 only simulated the interpolation of two identically shaped tensors. In reality, tensor swelling and FA diminishment due to interpolation involving tensors with different shapes and orientations in the three-dimensional space are expected to be even more severe. In such a case, use of the more sophisticated rotationally linear interpolation would be necessary to retain possible group differences in FA. At least for the subjects included in our study, rotationally linear interpolation seems to be satisfactory in effectively preserving the group FA behaviors.

In summary, the findings in both VBS and ROI analyses as well as computer simulations support our hypothesis that the different interpolation approaches used for DTI spatial normalization could potentially affect the results and interpretation of group comparison. Disappearance of group difference in FA in linear interpolation could likely occur in regions with a substantial amount of crossing fibers, a finding that is further corroborated by computer simulations.

One minor issue that was not answered in this study is the overall FA decrease (leftward shift of the FA histogram) after spatial normalization no matter which interpolation algorithm was used. While the computer simulation in Fig. 8 provides a valid explanation that interpolation of tensors with different shapes could lead to FA reduction, whether this FA reduction would result in a vanishing of group difference remains to be shown. Nevertheless, the FA reduction effect due to shape difference seems to be comparably much less than that due to, for example, orientation difference in linear interpolation (Fig. 7). Another issue that has not been answered by our study is the reversed effect. That is, although we have only shown that spatial normalization could obscure a true FA difference, the possibility that normalization might also create an artificial difference cannot be ruled out. For example, in the RACR, if linear interpolation caused a greater FA decrease in healthy control subjects than in ADHD patients, the ADHD patients would appear to have a higher FA value compared with the healthy control subjects if the true FA had been identical. Even if this was not observed in our study, careful attention should be paid in the interpretation of group results.

It may be argued that simply using ROI analysis without application of any spatial normalization algorithm would suffice in measuring FA. However, ROIs cannot be decided without a priori knowledge of the target tissue involved, particularly when no visually noticeable abnormality is present and no precise pathophysiology is known in the majority of psychiatric disorders. The ROI method has the strength of anatomical validity, but rigorous training is needed to ensure rater reliability. This means that novel image analytical methods not requiring prior selection of ROIs, such as automated VBS analysis, would be needed at least in research filed for hypothesis generation. Hence, the procedure of spatial normalization remains to be an essential step, and the associated methodologies involved in DTI spatial normalization should be surveyed to determine their merit and consistency. In addition, it should be noted that FA is only one of the DTI indices in exploring white matter microstructural integrity. Given the fact that, as this study reported, FA alteration was present after applying algorithms already dedicated to have optimal preservations of the diffusion tensor characteristics [14-19], large-scale DTI studies would be needed to further explore the possible effects of spatial normalization on other DTI indices such as the principal eigenvector and directional diffusivities [28]. Another possibility to circumvent the issues of DTI normalization is to perform scalar spatial normalization directly on the original EPI images. While this approach avoids the demands of technical developments, we did not choose this method in our study because the computational load involved in the case of our 15-direction DTI protocol could be greater.

Finally, we have no intention to make conclusive interpretations about the FA findings of ADHD in this 
study due to the limited sample size. The purpose of this article is to demonstrate possible technical influences on DTI indices, namely, the effects of interpolation in spatial normalization on FA values. Overall, our results provide valuable information to guide future DTI studies by reporting that the interpolation procedure may obscure possible group differences in FA. Both human imaging data and computer simulations suggest the phenomena to be worthy of special attention in areas of crossing fibers, such as the corona radiata. Spatial normalization of DTI data should therefore be executed with care even when software algorithms dedicated to have optimal preservations of the DTI characteristics are used. Our study has important implications for the investigations of white matter microstructural integrity in psychiatric disorders in which VBS DTI analysis for group comparison is used increasingly.

\section{Acknowledgment}

This study was supported in part by the National Science Council under grants NSC95-2314-B-002-221-MY3 and NSC95-2314-B-037-098-MY2.

\section{References}

[1] Dong Q, Welsh RC, Chenevert TL, Carlos RC, Maly-Sundgren P, Gomez-Hassan DM, et al. Clinical applications of diffusion tensor imaging. J Magn Reson Imaging 2004;19:6-18.

[2] Freedman J. The diffusion of innovations into psychiatric practice. Psychiatr Serv 2002;53:1539-40.

[3] Lim KO, Helpern JA. Neuropsychiatric applications of DTI - a review. NMR Biomed 2002;15:587-93.

[4] Sotak $\mathrm{CH}$. The role of diffusion tensor imaging in the evaluation of ischemic brain injury - a review. NMR Biomed 2002;15:561-9.

[5] Abe O, Yamada H, Masutani Y, Aoki S, Kunimatsu A, Yamasue H, et al. Amyotrophic lateral sclerosis: diffusion tensor tractography and voxel-based analysis. NMR Biomed 2004;17:411-6.

[6] Park HJ, Westin CF, Kubicki M, Maier SE, Niznikiewicz M, Baer A, et al. White matter hemisphere asymmetries in healthy subjects and in schizophrenia: a diffusion tensor MRI study. Neuroimage 2004;23:213-23.

[7] Ashburner J, Friston KJ. Why voxel-based morphometry should be used. Neuroimage 2001;14:1238-43.

[8] Ashburner J, Andersson JL, Friston KJ. Image registration using a symmetric prior — in three dimensions. Hum Brain Mapp 2000;9:212-25.

[9] Ashburner J, Friston KJ. Nonlinear spatial normalization using basis functions. Hum Brain Mapp 1999;7:254-66.

[10] Good CD, Johnsrude IS, Ashburner J, Henson RN, Friston KJ, Frackowiak RS. A voxel-based morphometric study of ageing in 465 normal adult human brains. Neuroimage 2001;14:21-36.
[11] Burns J, Job D, Bastin ME, Whalley H, Macgillivray T, Johnstone EC, et al. Structural disconnectivity in schizophrenia: a diffusion tensor magnetic resonance imaging study. Br J Psychiatry 2003;182:439-43.

[12] Eriksson SH, Rugg-Gunn FJ, Symms MR, Barker GJ, Duncan JS. Diffusion tensor imaging in patients with epilepsy and malformations of cortical development. Brain 2001;124:617-26.

[13] Foong J, Symms MR, Barker GJ, Maier M, Miller DH, Ron MA. Investigating regional white matter in schizophrenia using diffusion tensor imaging. Neuroreport 2002;13:333-6.

[14] Jones DK, Griffin LD, Alexander DC, Catani M, Horsfield MA, Howard R, et al. Spatial normalization and averaging of diffusion tensor MRI data sets. Neuroimage 2002;17:592-617.

[15] Park HJ, Kubicki M, Shenton ME, Guimond A, McCarley RW, Maier SE, et al. Spatial normalization of diffusion tensor MRI using multiple channels. Neuroimage 2003;20:1995-2009.

[16] Xu D, Mori S, Shen D, van Zijl PC, Davatzikos C. Spatial normalization of diffusion tensor fields. Magn Reson Med 2003;50: $175-82$.

[17] Alexander DC, Pierpaoli C, Basser PJ, Gee JC. Spatial transformations of diffusion tensor magnetic resonance images. IEEE Trans Med Imaging 2001;20:1131-9.

[18] Arsigny V, Fillard P, Pennec X, Ayache N. Log-Euclidean metrics for fast and simple calculus on diffusion tensors. Magn Reson Med 2006; 56:411-21.

[19] Batchelor PG, Moakher M, Atkinson D, Calamante F, Connelly A. A rigorous framework for diffusion tensor calculus. Magn Reson Med 2005;53:221-5.

[20] Puig-Antich J, Chambers W. The Schedule for Affective Disorders and Schizophrenia for School-Age Children (Kiddie-SADS). New York: New York State Psychiatric Institute; 1978.

[21] Reese TG, Heid O, Weisskoff RM, Wedeen VJ. Reduction of eddycurrent-induced distortion in diffusion MRI using a twice-refocused spin echo. Magn Reson Med 2003;49:177-82.

[22] Mangin JF, Poupon C, Clark C, Le Bihan D, Bloch I. Distortion correction and robust tensor estimation for MR diffusion imaging. Med Image Anal 2002;6:191-8.

[23] Mistry NN, Hsu EW. Retrospective distortion correction for 3D MR diffusion tensor microscopy using mutual information and Fourier deformations. Magn Reson Med 2006;56:310-6.

[24] Jones DK, Symms MR, Cercignani M, Howard RJ. The effect of filter size on VBM analyses of DT-MRI data. Neuroimage 2005;26:546-54.

[25] Snook L, Plewes C, Beaulieu C. Voxel based versus region of interest analysis in diffusion tensor imaging of neurodevelopment. Neuroimage 2007;34:243-52.

[26] Mascalchi M, Tessa C, Moretti M, Della Nave R, Boddi V, Martini S, et al. Whole brain apparent diffusion coefficient histogram: a new tool for evaluation of leukoaraiosis. J Magn Reson Imaging 2002;15:144-8.

[27] Rovaris M, Iannucci G, Cercignani M, Sormani MP, De Stefano N, Gerevini S, et al. Age-related changes in conventional, magnetization transfer, and diffusion-tensor MR imaging findings: study with wholebrain tissue histogram analysis. Radiology 2003;227:731-8.

[28] Budde MD, Kim JH, Liang HF, Schmidt RE, Russell JH, Cross AH, et al. Toward accurate diagnosis of white matter pathology using diffusion tensor imaging. Magn Reson Med 2007;57:688-95. 